\title{
Unraveling Exciton Kinetics of Electroluminescence in Colloidal Quantum Dot LEDs
}

\author{
Sushant Shendre ${ }^{1}$, Cuong Dang*1, Hilmi Volkan Demir*1,2 \\ ${ }^{1}$ LUMINOUS! Centre of Excellence for Semiconductor Lighting and Displays, School of Electrical and Electronic Engineering, \\ and School of Physical and Mathematical Sciences, Nanyang Technological University, Nanyang Avenue, Singapore 639798, \\ ${ }^{2}$ Department of Electrical and Electronics Engineering and Department of Physics, UNAM-Institute of Materials Science and \\ Nanotechnology, Bilkent University, Bilkent, Ankara, Turkey 06800. \\ Email: HVDEMIR@ntu.edu.sg and HCDang@ntu.edu.sg,
}

\begin{abstract}
We systematically studied emission kinetics of colloidal quantum dots in active light-emitting diodes using time-correlated single photon counting and conclusively revealed the combined effect of both electric field and charging acting together in reducing the quantum efficiency during operation.

OCIS codes: (250.5590) Quantum-well, -wire and -dot devices, (230.0250) Optoelectronics, (160.4236) Nanomaterials
\end{abstract}

\section{Introduction}

The study of colloidal quantum dot light-emitting diodes (QD-LEDs) has made a major progress to achieve high efficiency devices $[1,2]$ for lighting and display technologies. Subsequent research work [3-5] investigating the causes of efficiency droop in these devices showed the effect of electric field or charging independently as the primary causes for specific devices. Our hypothesis is that the two effects co-exist in most operating QD-LEDs, and here we show and analyze the simultaneous effect of both electric field and charging.

\section{Experiment and Results}

Using our own synthesized CdSe@ZnS QDs having 70\% quantum yield (QY) in solution, 30\% in film (measured with integrating sphere), we fabricated devices by spin-coating and vacuum deposition of layers in the following architectures: capacitor device (Figure 1a) comprising ITO/Poly(methyl methacrylate) (PMMA,100nm)/QDs(50nm) $/ \mathrm{PMMA}(100 \mathrm{~nm}) / \mathrm{Al}(>100 \mathrm{~nm})$ and LED device (Figure 1b) consisting of ITO/ZnO(50nm)/QDs(30nm)/4,4'-Bis(9-carbazolyl)-1,1'-biphenyl (CBP,60nm)/ $\mathrm{MoO}_{3}(10 \mathrm{~nm}) / \mathrm{Al}(>100 \mathrm{~nm})$.

We measured the time resolved photoluminescence (PL) decay for both the devices under different bias and fit the curves using a twocomponent exponential decay fit, $I(t)=A_{1} \exp \left(-t / \tau_{1}\right)+A_{2} \exp \left(-t / \tau_{2}\right)$. The obtained lifetimes $\left(\tau_{1}, \tau_{2}\right)$ and the photon count associated with each population $\left(A_{i} \tau_{i}\right.$, where $\left.i=1,2\right)$ obtained by integrating the area under the decay curves are presented in Figure 2 for the capacitor device and the LED. We associate the lifetimes to two populations of QDs (Population \#1 and \#2) with the Population 1 lifetime having the smaller value and 2, the larger. We believe these two populations arise due to the formation of two types of QDs in the film, those which are near to the adjacent layers and the ones deep within the QD film away from the adjacent layers. Since each lifetime $\left(\tau_{\mathrm{i}}\right)$ is composed of radiative $\left(\tau_{\mathrm{ri}}\right)$ and nonradiative $\left(\tau_{\text {nri }}\right)$ parts related together as, $\left(1 / \tau_{\mathrm{i}}\right)=\left(1 / \tau_{\mathrm{ri}}\right)+\left(1 / \tau_{\text {nri }}\right)$, where $\mathrm{i}=1,2$ refer to Population 1 and 2, respectively; Population 1 (shorter) lifetime in each case has a higher proportion of nonradiative part $\left(\tau_{\text {nri }}\right)$. Also, with $30 \%$ QY of our ensemble QD film, there is a significant amount of QDs (Population 2) with relatively high radiative rate, we expect to see the effect of applied bias on the radiative component of lifetime in the variations of Population

a)

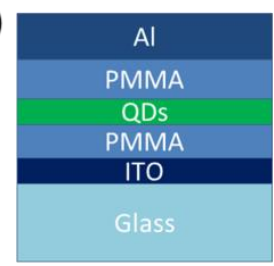

b)

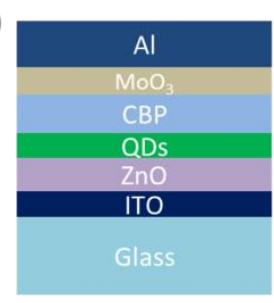

Figure 1. Schematic diagram a) Capacitor device, b) LED device 2 (longer) lifetime.

As can be seen in the capacitor device (Figure 2a), increasing the electric field above zero causes Population 2 (longer) lifetime to increase from 7.9 to 9.3 ns between the extremities. This can be explained with the increasing of radiative lifetime under electric field similar to [4]. Since Population 1 (shorter) lifetime (Figure 2a) does not show any systematic variation with field, it is difficult to infer the effect of field on the nonradiative lifetime of our QDs.

Using a similar analysis, for LED device (Figure 2b), Population 2 lifetime increases from 5.0 to $5.6 \mathrm{~ns}$ when the applied bias is increased from 0 to $-5 \mathrm{~V}$. It can be concluded that under reverse bias where the current density is low, the QDs in the LED behave in a similar way to the capacitor device. 
In the forward bias, Population 2 lifetime increases to around $5.3 \mathrm{~ns}$ on increasing bias up to $3 \mathrm{~V}$ and then down to $4.3 \mathrm{~ns}$ when the bias is increased further to $4.0 \mathrm{~V}$. Under low current density till $3.0 \mathrm{~V}$, QDs behave similar to the reverse bias conditions. Once the current density rises rapidly, the lifetime falls possibly due to the increase in nonradiative rate. It is indeed the case when we observe the lifetime of Population 1, which has predominant nonradiative component, the lifetime decreases from around $1.7 \mathrm{~ns}$ at $1.5 \mathrm{~V}$ to $1.0 \mathrm{~ns}$ at $4.0 \mathrm{~V}$. This can be attributed to the charging of the QDs due to increasing current density leading to enhancement of Auger recombination rate. It is to be noted that the LED starts electroluminescence from $3 \mathrm{~V}$ and onwards. Thus, it can be inferred that, under low current densities, the electric field effect is predominant, while at higher current density levels, the effect of charging takes precedence.

From Figures $2 \mathrm{c}$ and $2 \mathrm{~d}$, since the major proportions of photons are emitted by Population 2 in both devices, it is the population with higher quantum efficiency. Here we see that both the electric field and charging leads to a reduction in the quantum efficiency of the QDs.
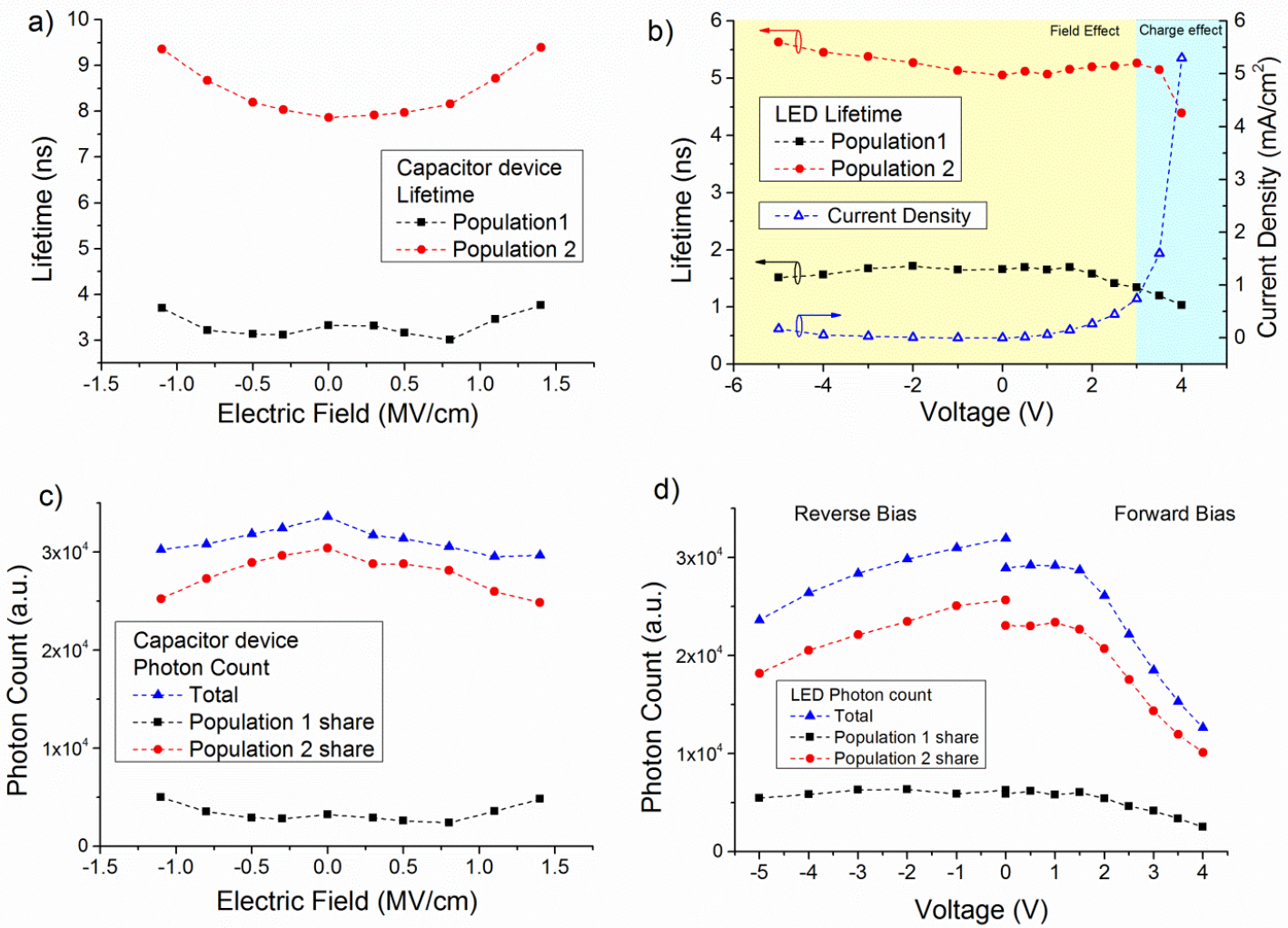

Figure 2. Variation of PL decay lifetimes of two populations obtained from two-component exponential fit of the decay curves in a) capacitor device and b) LED device. Variation of photon count with bias for each population and their total, obtained from integrating the area below the decay curves for c) capacitor device and d) LED device.

\section{Conclusion}

By systematically studying the time-resolved emission of the quantum dots layer in the active device under different biases, we observe two opposite behaviors of exciton recombination processes leading to increasing radiative lifetime with respect to the electric field and decreasing nonradiative lifetime with respect to the charging. Both these effects cause reduction in quantum efficiency of the QDs.

\section{References}

[1] B. S. Mashford, M. Stevenson, Z. Popovic, C. Hamilton, Z. Zhou, C. Breen, et al., "High-efficiency quantum-dot light-emitting devices with enhanced charge injection," Nature photonics, vol. 7, pp. 407-412, 2013.

[2] X. Dai, Z. Zhang, Y. Jin, Y. Niu, H. Cao, X. Liang, et al., "Solution-processed, high-performance light-emitting diodes based on quantum dots," Nature, 2014.

[3] Y. Shirasaki, G. J. Supran, W. A. Tisdale, and V. Bulović, "Origin of efficiency roll-off in colloidal quantum-dot light-emitting diodes," Physical review letters, vol. 110, p. 217403, 2013.

[4] D. Bozyigit, O. Yarema, and V. Wood, "Origins of low quantum efficiencies in quantum dot LEDs," Advanced Functional Materials, vol. 23, pp. 3024-3029, 2013.

[5] W. K. Bae, Y.-S. Park, J. Lim, D. Lee, L. A. Padilha, H. McDaniel, et al., "Controlling the influence of Auger recombination on the performance of quantum-dot light-emitting diodes," Nature communications, vol. 4, 2013. 